\title{
Real-time computer-generated hologram by means of liquid-crystal television spatial light modulator
}

\author{
Fai Mok \\ Department of Electrical Engineering, California Institute of Technology, Pasadena, California 91125 \\ Joseph Diep \\ Department of Optical Engineering, University of La Verne, La Verne, California 91750 \\ Hua-Kuang Liu \\ Jet Propulsion Laboratory, California Institute of Technology, Pasadena, California 91109 \\ Demetri Psaltis \\ Department of Electrical Engineering, California Institute of Technology, Pasadena, California 91125
}

Received March 17, 1986; accepted August 8, 1986

A novel use of liquid-crystal television (LCTV) is described. It is shown that, if the phase nonuniformity of the LCTV is corrected by a liquid gate, then a simple computer-generated hologram can be written and coherently reconstructed.

It is well known that spatial light modulators (SLM's) are essential for real-time optical information processing. For that reason, much research has been devoted to them, and many types of SLM have been developed. ${ }^{1}$

Most of the existing SLM's are either forbiddingly expensive or still in the research and development stages. Recently an extremely inexpensive liquidcrystal television (LCTV) was investigated in relation to its use in optical data processing. ${ }^{2-10}$ One advantage of the LCTV is that it can be addressed either electronically or optically. However, because of the thickness variations over the aperture of the screen of the devices, its usefulness for coherent optical processing is limited.

We have examined the thickness nonuniformities of the LCTV screen in a Mach-Zehnder interferometer. We have successfully corrected the nonuniformities by submerging the screen in a liquid gate and have demonstrated the feasibility of using this device to write and reconstruct a computer-generated hologram. The results are briefly reported below.

The operating principle of the LCTV has been described..$^{2,3}$ The device has a $5.4 \mathrm{~cm}$ by $4.4 \mathrm{~cm}$ screen that consists of a two-dimensional mosaic of individually addressable liquid-crystal cells that contain a 90deg twisted nematic liquid crystal sandwiched between two glass plates. These plates have, respectively, transparent horizontal-line and vertical-line electrodes deposited on their inner surfaces facing the liquid crystal. Two linear polarizer sheets (polarizers) are glued to the outer surfaces of the glass plates. The polarization axes of these polarizers are parallel.
When no electric field is applied, the plane of polarization for linearly polarized light is rotated through 90 deg by the twisted liquid-crystal molecules, and no light is transmitted through the second polarizer. However, with an electric field applied, the twist and tilt of the liquid-crystal molecules are altered, resulting in transmission of a controllable and variable fraction of light. The input signal to the LCTV can be from a TV receiver, a computer, or a TV camera.

A Mach-Zehnder interferometer was set up to analyze the optical quality of the LCTV screen. A 6.4$\mathrm{cm}^{2}$ (1-in. $\left.{ }^{2}\right)$ region of the LCTV screen was illuminated by a collimated $\mathrm{He}-\mathrm{Ne}$ laser beam. The results are shown in Fig. 1(a). It can be seen that more than five fringes are produced, indicating thickness nonuniformities of the LCTV screen amounting to about 0.046 $\mu \mathrm{m}$ per pixel. It has been found that the phase nonuniformity is due mainly to the poor quality of the polarizer sheets. Because of the nonuniformity, Fraunhofer diffraction patterns show fuzziness in the various orders. ${ }^{3}$

We investigated two ways of removing the phase distortions. The first was to correct the phase variation by a phase-conjugation method. We used a 10 $\mathrm{mW} \mathrm{He}-\mathrm{Ne}$ laser to produce a holographic filter of the LCTV screen and addressed it with a phase-conjugated beam projected through a holographic plate. Unfortunately, the beam reconstructed through the LCTV was too weak to be observed visually because of the low diffraction efficiency of the hologram and the low power of the laser. The effectiveness of the phaseconjugation technique was, therefore, not conclusively shown. However, Casasent and Xia have proved that 
this is a viable way of correcting the phase-nonuniformity problem. ${ }^{7}$

We then used a liquid-gate correction technique. The LCTV screen was submerged in a liquid gate (NRC Model 550) filled with an index-matching nonconductive mineral oil with a refractive index of about 1.45. In this case, the optical path length through the liquid gate was made nearly constant. Because it is hard to match simultaneously the different indices of the LCTV screen (glass plates plus polarizers), a small amount of phase variation remained. Nevertheless, we have found that most of the thickness variation of the TV screen was corrected. Figure 1(b) shows an interferogram of the liquid gate without the LCTV screen. Note that much less than one fringe appears across the illuminated region when the liquid gate (filled with mineral oil) is placed in the path of the Mach-Zehnder interferometer.

Figure 1(c) is an interferogram of the LCTV screen submerged in the same liquid gate.

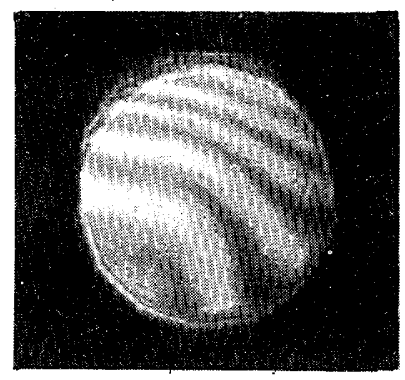

(a)

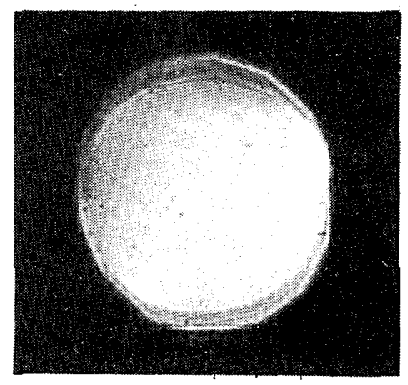

(b)

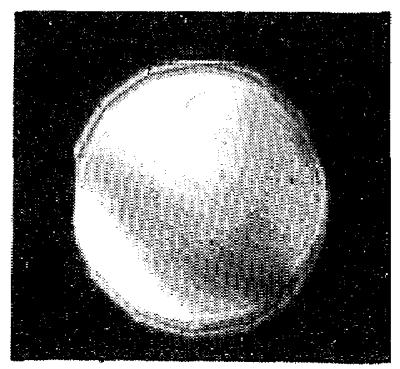

(c)

Fig. 1. Phase-nonuniformity correction of the LCTV in a Mach-Zehnder interferometer (MZI) as showm by (a) an interferogram with LCTV in a MZI, (b) an interferogram with liquid-filled liquid gate in a MZI, and (c) an interferogram with LCTV submerged in liquid gate in a MZI.

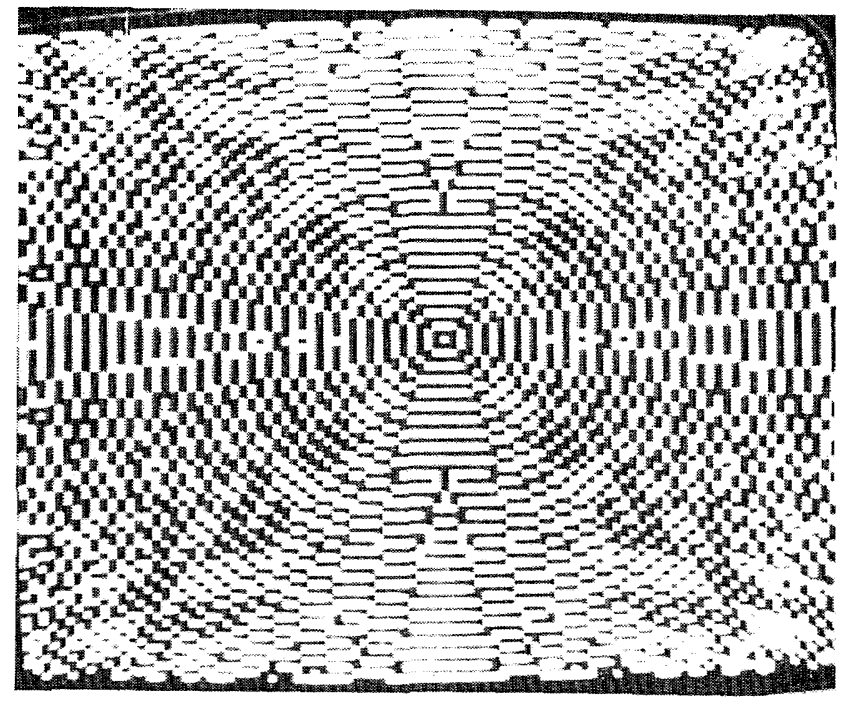

(a)

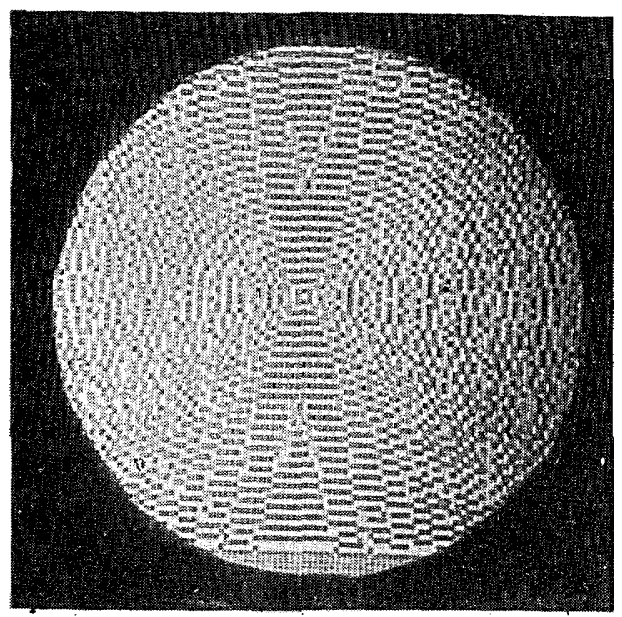

(b)

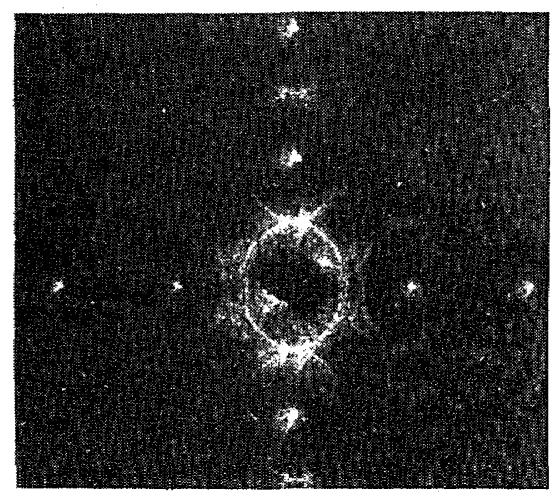

(c)

Fig. 2. Computer-generated hologram of a circle: (a) on a conventional TV monitor, (b) on LCTV; (c) coherent-optical reconstruction.

With the phase distortion of the LCTV mostly corrected, several coherent-optical data-processing experiments can be performed, including an optical matched-filtering experiment. ${ }^{3}$ We thought it interesting to try to write a computer-generated hologram 
on the LCTV and reconstruct it through coherent light. A $100 \times 100$ pixel binary phase kinoform ${ }^{11}$ of a circle was first generated using an IBM PC computer. Figure 2(a) shows the kinoform displayed on a conventional TV monitor. The kinoform was produced by binary encoding of the phase, while the amplitude was kept constant, at each pixel of the Fourier transform of a circle. Basically, this kinoform consisted of on-axis, phase-modulated patterns written into the liquidcrystal layer through the electrodes controlled by the computer output. When the collimated laser light was transmitted through the liquid-crystal layer, the phase of the light was modulated. The output analyzer-polarizer served the function of transforming the phase variations into amplitude variations. However, only the vertical resolution of the kinoform matched that of the LCTV exactly. The LCTV interpolated the missing horizontal points to form an image as shown in Fig. 2(b).

The LCTV, submerged in the oil-filled NRC liquid gate, was then placed in an optical Fourier-transform system. The resulting holographic reconstruction of the circle is shown in Fig. 2(c). As expected, the elliptical appearance of the circle is due to the nonunity aspect ratio of the high-resolution format of the IBM PC. A zero-order dc spot has been blocked for clearer image presentation. The high-intensity dc spot not shown here is believed to be caused by the nonconstant rotations of polarization of the pixels of the same logic level.

To show the necessity of using the liquid gate in the present work, we also recorded a binary phase hologram of the letter $\mathrm{O}$ on a piece of film and placed it in front of the LCTV screen with the TV electronics turned off. The reconstruction of this composite filter was fuzzy. After the LCTV was removed, the reconstruction became well defined. Hence we may. conclude that the unimproved LCTV can introduce enough phase distortion to cause failure in reconstruction.

The results described in this Letter demonstrate the usefulness of an inexpensive LCTV as a spatial light modulator for coherent-optical processing in the writing and reconstruction of a single computer-generated hologram. Some similar results were achieved by
Psaltis et al., who used a magneto-optics device for reconstruction of a hologram. ${ }^{12}$ These findings should make it much easier and less expensive for many optics laboratories to conduct experiments on coherent and incoherent optical processing.

We are currently investigating the use of a largememory correlator ${ }^{13}$ with the LCTV as a real-time incoherent-to-coherent transducer and with its zero order suppressed.

The research described in this Letter was carried out by the Jet Propulsion Laboratory, California Institute of Technology, and was sponsored by the Physics Division of the U.S. Army Research Office and the National Aeronautics and Space Administration.

We would like to thank J. W. Goodman of Stanford University and A. Johnston of Jet Propulsion Laboratory for helpful discussion on the use of liquid in the liquid gate.

J. Diep is an academic part-time employee at Jet Propulsion Laboratory, California Institute of Technology.

\section{References}

1. G. R. Knight, in Optical Information Processing, S. H. Lee, ed., Vol. 48 of Topics in Applied Physics (SpringerVerlag, Berlin, 1981).

2. J. A. McEwan, A. D. Fisher, P. B. Rolsma, and J. N. Lee, J. Opt. Soc. Am. A 2(13), P8 (1985).

3. H. K. Liu, J. A. Davis, R. A. Lilly, Opt. Lett. 10, 635 (1985).

4. D. A. Gregory, Appl. Opt. 25, 467 (1986).

5. M. Young, Appl. Opt. 25, 1024 (1986).

6. A. M. Tai, Appl. Opt. 25, 1380 (1986).

7. D. Casasent and S. F. Xia, Opt. Lett. 11, 398 (1986).

8. H. K. Liu, S. Y. Kung, and J. Davis, Opt. Eng. 25, 853 (1986).

9. F. T. S. Yu, J. Jutamulia, and X. L. Huang, Appl. Opt. 25, 3324 (1986).

10. J. A. Davis, R. A. Lilly, K. D. Kreng, and H. K. Liu, Proc. Soc. Photo-Opt. Instrum. Eng. 613, 245 (1986).

11. L. B. Lesem, P. Hirsch, and J. A. Jordan, Jr., IBM J. Res. Dev. 13, 150 (1969).

12. D. Psaltis, E. G. Paek, and S. S. Venkatesh, Opt. Eng. 23, 698 (1984).

13. D. Gregory and H. K. Liu, Appl. Opt. 23, 4560 (1984). 